\title{
Structural phase transitions in epitaxial perovskite films
}

\author{
Feizhou He* and B. O. Wells \\ Department of Physics, University of Connecticut, Storrs, Connecticut 06269 \\ Z. -G. Ban and S. P. Alpay \\ Department of Materials Science and Engineering and Institute of Materials Science, \\ University of Connecticut, Storrs, Connecticut 06269 \\ S. Grenier, S. M. Shapiro, and Weidong Si \\ Department of Physics, Brookhaven National Lab, Upton, New York 11973
}

A. Clark and X. X. Xi
Department of Physics, The Pennsylvania State University, University Park, Pennsylvania 16802

(Dated: August 30, 2018)

\begin{abstract}
Three different film systems have been systematically investigated to understand the effects of strain and substrate constraint on the phase transitions of perovskite films. In $\mathrm{SrTiO}_{3}$ films, the phase transition temperature $\mathrm{T}_{C}$ was determined by monitoring the superlattice peaks associated with rotations of $\mathrm{TiO}_{6}$ octahedra. It is found that $\mathrm{T}_{C}$ depends on both $\mathrm{SrTiO}_{3}$ film thickness and $\mathrm{SrRuO}_{3}$ buffer layer thickness. However, lattice parameter measurements showed no sign of the phase transitions, indicating that the tetragonality of the $\mathrm{SrTiO}_{3}$ unit cells was no longer a good order parameter. This signals a change in the nature of this phase transition, the internal degree of freedom is decoupled from the external degree of freedom. The phase transitions occur even without lattice relaxation through domain formation. In $\mathrm{NdNiO}_{3}$ thin films, it is found that the in-plane lattice parameters were clamped by the substrate, while out-of-plane lattice constant varied to accommodate the volume change across the phase transition. This shows that substrate constraint is an important parameter for epitaxial film systems, and is responsible for the suppression of external structural change in $\mathrm{SrTiO}_{3}$ and $\mathrm{NdNiO}_{3}$ films. However, in $\mathrm{SrRuO}_{3}$ films we observed domain formation at elevated temperature through $\mathrm{x}$-ray reciprocal space mapping. This indicated that internal strain energy within films also played an important role, and may dominate in some film systems. The final strain states within epitaxial films were the result of competition between multiple mechanisms and may not be described by a single parameter.
\end{abstract}

PACS numbers: 68.18.Jk, 68.55.Jk, 77.55.+f, 77.80.-e

\section{INTRODUCTION}

Perovskite thin films have received great interest in recent years due to a variety of interesting properties such as high- $\mathrm{T}_{C}$ superconductivity, colossal magnetoresistivity (CMR), ferroelectricity and metal-insulator transition. While bulk crystals of perovskites have been well studied, thin films are not understood in nearly the same details. It is well known that the properties of thin films can be quite different from corresponding bulk materials. The reason is often believed to be the strain and high concentration of defects in the thin films. This work illustrates that another important parameter for films is the geometrical constraint imposed by the substrates. Due to the excellent epitaxy, the films are tied to the underlying substrate, therefore the in-plane lattice parameters of films are not free to attain their bulk equilibrium values. The macroscopic sizes of films are always the same as that of substrates This is known as the substrate clamping effect, or substrate constraint, which forces the temperature dependence of in-plane lattice parameters of films to follow that of substrates. This effect is often neglected but as discussed in this paper, it has great effect on phase transitions and domain formation in films. The strain and substrate constraint give us a method to fine-tune the properties of the thin films, or even create a new phase that is not possible in bulk form. In recent years there are emerging theoretical efforts to treat the effects of strain,, 2.3 .4 based on thermodynamic analysis using Landau theory of phase transition.

$\mathrm{SrTiO}_{3}$ (STO) has long been an important model system for condensed matter physics. More recently it has received attention for having a large and variable dielectric constant, making it ideal for tunable microwave devices. $\stackrel{5}{=}$ Bulk STO crystals are cubic perovskite at room temperature, with space group $\operatorname{Pm} \overline{3} \mathrm{~m}\left(\mathrm{O}_{h}^{1}\right)$, but become tetragonal, space group I4/mcm $\left(\mathrm{D}_{4 h}^{18}\right)$, below $105 \mathrm{~K}$. This phase transition was extensively studied in the 1960's and 1970's as the prototypical example of a soft-mode phase transition,,$\frac{6}{}$ involving a progressive softening of the zone boundary phonon mode due to the $\mathrm{TiO}_{6}$ octahedra rotation about a formerly cubic axis. When the energy for this mode at the zone boundary reaches zero, the lower symmetry structure condenses. Since the phonon condensation occurs at the zone boundary, this phase transition is non-polar and antiferrodistortive. The high temperature structure of $\mathrm{SrTiO}_{3}$ is the same as that of $\mathrm{BaTiO}_{3}$ (BTO), the canonical ferroelectric crystal. In 
BTO, the ferroelectric phase transition is associated with a softening of a zone-center phonon mode in which the central $\mathrm{Ti}$ ion shifts with respect to the oxygen cage. For $\mathrm{SrTiO}_{3}$, the ferroelectric phase, which almost occurs at lower temperatures, is suppressed by quantum fluctuations. ${ }^{7}$ However the ferroelectric state can be in-

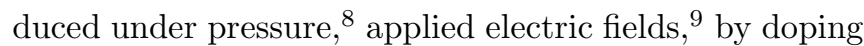

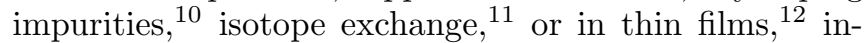
dicating that this phase transition is sensitive to lattice strain. Therefore, studying the properties of perovskite films under strain conditions may as well help to better understand the ferroelectric phase transitions in related systems, such as $\mathrm{BaTiO}_{3}$ and $\mathrm{PbTiO}_{3}$. In fact, similar structural phase transitions occur in most perovskitebased oxide crystals, for example $\mathrm{SrRuO}_{3}$. Therefore, the results we obtained here may be generalized to other perovskite films systems.

$\mathrm{SrRuO}_{3}$ (SRO) is a ferromagnetic perovskite with Curie temperature of about 160K. It has excellent electrical conductivity and is chemically stable. Since SRO has good lattice matching with other perovskites, it is the material of choice for electrodes of perovskite-based devices. However, a bottom SRO layer may change the strain state and thus the properties of the top layer. A detailed study on effects of buffer layers is necessary, so that we can either minimize the negative influence, or utilize the effect to tune the strain state of the layers above. Here we investigated the influence of SRO buffer layers on the phase transition of STO films, and the domain structures of SRO layers.

We also studied the behavior of $\mathrm{NdNiO}_{3}(\mathrm{NNO})$ thin films under biaxial strain. Perovskite nickelates $\left(\mathrm{RNiO}_{3}\right.$ with $\mathrm{R}=$ rare earth) show metal-insulator (MI) transitions with the transition temperature rising systematically as the size of the rare earth decreases, which implies an increase in the distortion away from the ideal cubic perovskite. This transition is first-order in nature and associated with volume change across the phase transition. Bulk NNO has shown large pressure dependence of the MI transition, ${ }^{13}$ with change in $\mathrm{T}_{C}$ of over $100 \mathrm{~K}$ under high pressure. Similar effects have been observed

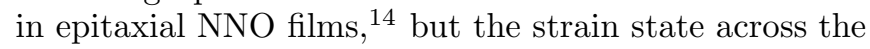
MI transition and the microstructure of NNO films are not clear.

In earlier work, we reported experimental results of the strain effects on the phase transitions of STO thin films, and the observations of the smooth evolution of the outof-plane lattice parameters across the transition. ${ }^{15}$ A theoretical analysis has also been undertaken to understand the strain relaxation mechanism in epitaxial STO films and the influence of the SRO buffer layers ${ }^{16}$ In this paper, a systematic study of the misfit strain and substrate clamping effects on phase transitions of perovskite films is reported. We show that the thickness of both thin films and buffer layers may influence $\mathrm{T}_{C}$, and the temperature dependence of the lattice relaxation is determined by the substrate clamping effect and the volume change associated with the phase transition. The mechanisms involved in determining the final strain states in films are discussed.

\section{EXPERIMENT}

The STO films were grown by pulsed laser deposition (PLD) on $\mathrm{LaAlO}_{3}$ (LAO) single crystal substrates. Before growing the STO films, $\mathrm{SrRuO}_{3}$ buffer layers of various thickness were deposited. The SRO buffer serves as both a method to tune the strain state in the STO films and as a base electrode for electrical measurements. The samples are of the STO/SRO/LAO type, with STO film thickness range from $10 \mathrm{~nm}$ to $1 \mu \mathrm{m}$ and SRO buffer layers from $0 \mathrm{~nm}$ to $350 \mathrm{~nm}$. Details of the sample preparation are available elsewhere 17 We report on experiments of both the STO and SRO layers.

The $\mathrm{NdNiO}_{3}$ thin films, with thickness of $60 \mathrm{~nm}$ and $200 \mathrm{~nm}$, were grown on LAO substrates by PLD. A KrF excimer laser was used with an energy density of $\sim 2.0$ $\mathrm{J} / \mathrm{cm}^{2}$ and a repetition rate of $5 \mathrm{~Hz}$. The substrate was heated to $700^{\circ} \mathrm{C}$ and an oxygen pressure of $200 \mathrm{mTorr}$ was used during the deposition. After deposition, the films were cooled to room temperature at a rate of $60^{\circ} \mathrm{C}$ per minute. Resistivity was measured by the standard four probe method through $5 \mathrm{~K}$ to $300 \mathrm{~K}$ with current about $0.5 \mu \mathrm{A}$.

$\mathrm{X}$-ray diffraction (XRD) measurements were carried out at beamline X22A and X22C at the National Synchrotron Light Source, Brookhaven National Laboratory. Synchrotron radiation has the advantages of high intensity and small divergence angle, which are critical for studying very thin films grown on substrates and buffers of similar structures. X22A has a bent $\mathrm{Si}(111)$ monochromator, giving a small beam spot and a fixed incident photon energy of $10 \mathrm{keV}$. The longitudinal resolution with a $\mathrm{Si}(111)$ analyzer was at least $0.001 \AA^{-1}$ (HWHM) for an (002) peak, as measured from the substrate. X22C has a $\mathrm{Ge}(111)$ double monochromator, focused in vertical plane, with variable energy range $3 \sim 12 \mathrm{keV}$. The samples ware cooled in a closed cycle refrigerator with a temperature control better than $\pm 0.5 \mathrm{~K}$. Above room temperature the sample was heated in a high temperature capable displex with a base temperature near $10 \mathrm{~K}$ and a maximum of $800 \mathrm{~K}$.

Throughout this paper we list most peaks with reference to a cubic cell, unless otherwise specified. For cubic notations, we define the axis normal to the surface of the substrate is the $c$ axis. Thus our scattering peaks, and reciprocal lattice positions, are listed as $(h k l)$ with $l$ the reciprocal direction perpendicular to the surface. For tetragonal or orthorhombic phases, a subscript $t$ or $o$ is added to the peak index, and the orientation is redefined accordingly. 


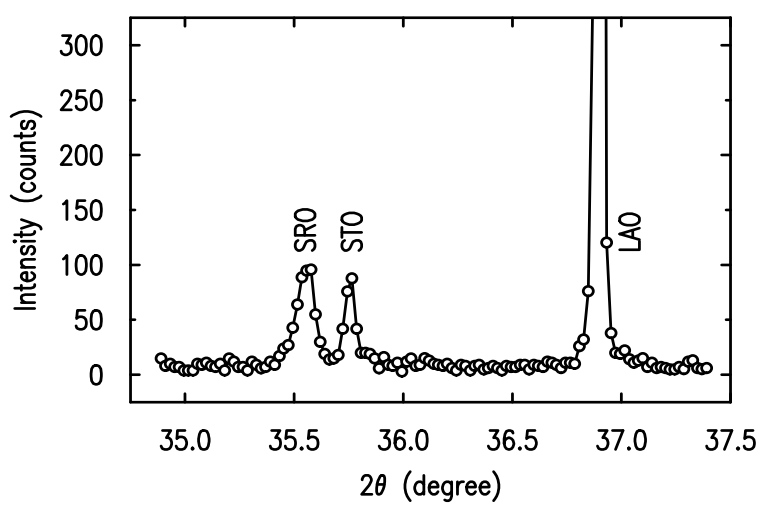

FIG. 1: Raw XRD scan for ( $\left.\begin{array}{lll}0 & 0 & 2\end{array}\right)$ peaks on $200 \mathrm{~nm}$ STO / $350 \mathrm{~nm}$ SRO / LAO sample, showing well resolved peaks. Photon energy is $10 \mathrm{keV}$.

\section{RESULTS}

\section{A. $\mathrm{SrTiO}_{3}$}

The high intensity and the collimation of the synchrotron x-ray source allows for scattering from very thin films in a high resolution mode to easily separate filmbuffer-substrate peaks. A typical scan over the $\left(\begin{array}{lll}0 & 0 & 2\end{array}\right)$ peaks is shown in Fig. 1 The STO and SRO peaks are well resolved, moreover the bulk LAO peak can be used as an internal reference to minimize systematic error. Xray diffraction and TEM (not shown here) show that all the thin films are in excellent epitaxy with the LAO substrates and SRO buffer layers.

\section{The Phase Transition}

In bulk STO, the soft mode phase transition occurs at $105 \mathrm{~K}$. This structural phase transition from cubic to tetragonal symmetry involves the rotation of the $\mathrm{TiO}_{6}$ octahedra around [ $\left[\begin{array}{lll}0 & 0 & 1\end{array}\right]$ axis. Because the $\mathrm{TiO}_{6}$ octahedra share the corner oxygen atoms, the neighboring $\mathrm{TiO}_{6}$ rotate in opposite directions. This leads to a tetragonal lattice with space group $\mathrm{I} 4 / \mathrm{mcm}$. The unit cell of the tetragonal phase is about $\sqrt{2} a \times \sqrt{2} a \times 2 a$, where $a$ is the lattice parameter of original cubic unit cell, therefore the volume of the tetragonal unit cell is about four times as that of the cubic unit cell. This tetragonal phase is a superlattice of the original cubic phase. The selection rule of the diffraction peaks is (in tetragonal notation): $h k l: h+k+l=2 n ; \quad 0 k l: l=2 n ; \quad h 0 l: l=2 n ;$

$h h l: l=2 n ; \quad h \bar{h} l: l=2 n$

In pseudo-cubic notation, the additional superlattice peaks are at half integer positions, such as $(1 / 2,1 / 2$, $3 / 2)_{c}$.

In STO films, we also observed the appearance of the superlattice peaks below $\mathrm{T}_{C}$, as shown in Fig. 2] which indicates that the phase transition also occurs in thin films. The temperature dependence of the peak inten-

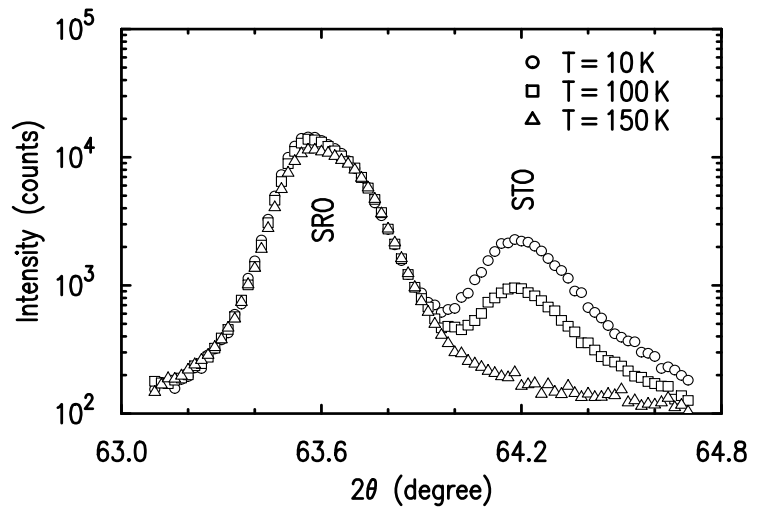

FIG. 2: Temperature dependence of $(1 / 2,1 / 2,7 / 2)$ STO superlattice peak for $1 \mu \mathrm{m}$ STO / $350 \mathrm{~nm}$ SRO / LAO sample. The other peak is from SRO layer.
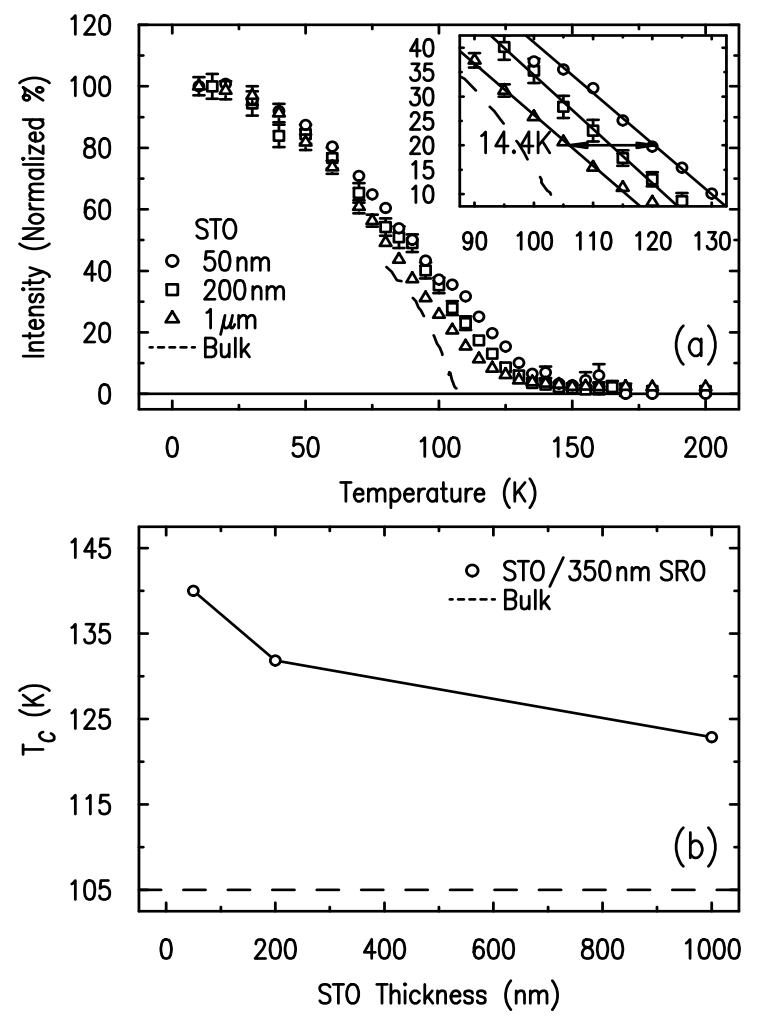

FIG. 3: Temperature dependence of superlattice peak intensity on STO film thickness. Bulk value from Ref. 19.

sity, as seen in Fig. 3 shows obvious rounded tails around $\mathrm{T}_{C}$, which means the phase transitions occur over a range of temperature. This is considered normal for films due to strain and defects, but it makes determination of $\mathrm{T}_{C}$ more difficult. We chose to linearly extrapolate the part of curves between $10 \%$ and $40 \%$ of maximum intensity, and define the $\mathrm{T}_{C}$ as where this line crosses the $0 \%$ line. This method is justified by both our data as well as in literature ${ }^{18}$ Though it might give a $\mathrm{T}_{C}$ slightly higher than the true transition, we believe the comparison between different films is accurate. 
Our first set of samples have STO film thickness varying from $50 \mathrm{~nm}$ to $1 \mu \mathrm{m}$, with the same SRO buffer layer thickness of $350 \mathrm{~nm}$. This shows us how the phase transition depends on the thickness of STO films, as other conditions keep the same. The $\mathrm{T}_{C}$ difference, $\Delta \mathrm{T}_{C}$, is about $14 \mathrm{~K}$ between the $50 \mathrm{~nm}$ sample and $1000 \mathrm{~nm}$ sample, as seen in Fig. 3. The thinner film has higher $\mathrm{T}_{C}$, while thicker film has $\mathrm{T}_{C}$ closer to the bulk value. The in-plane tensile strain measured by XRD at room temperature increases from $0.01 \%$ in $1000 \mathrm{~nm}$ film to $0.23 \%$ in $50 \mathrm{~nm}$ film. Therefore in this case, $\mathrm{T}_{C}$ increases monotonically with increasing in-plane strain.

The second set of samples have the same STO thickness of $200 \mathrm{~nm}$, but SRO thickness ranges from $0 \mathrm{~nm}$ to 350 $\mathrm{nm}$. This gives us information about the influence of the SRO buffer layers. We found that varying the SRO thickness has even bigger effect on $\mathrm{T}_{C}$, as shown in Fig 4 The thinnest $(5 \mathrm{~nm}) \mathrm{SRO}$ layer causes the highest $\mathrm{T}_{C}$ in $\mathrm{STO}$ films, which is about $50 \mathrm{~K}$ above the bulk value. When SRO thickness increases, $\mathrm{T}_{C}$ decreases. However, room temperature XRD measurement shows that the in-plane strains in these samples are still quite small, with the largest one only about $0.25 \%$, so this amount of change in $\mathrm{T}_{C}$ is fairly remarkable. It is interesting to notice that with SRO thickness decreased to $0 \mathrm{~nm}$, i.e., STO films grown directly on LAO substrate, the $\mathrm{T}_{C}$ goes back to about $130 \mathrm{~K}$. This signals that the SRO layers have a significant effect on the strain states of STO films.

\section{Lattice Parameters}

The lattice constants were measured by conventional diffraction as well as grazing incidence X-ray diffraction (GIXD) technique. Due to the high intensity of the synchrotron radiation, peaks from all three layers, even as thin as $20 \mathrm{~nm}$, can be seen clearly. Measuring multiple peaks in $\left(\begin{array}{lll}0 & 0 & l\end{array}\right)$ direction gives a very accurate measurement of the $c$ lattice constant. In-plane lattice constants are obtained either by measuring a partially inplane peak such as $\left(\begin{array}{lll}2 & 0 & 2\end{array}\right) /\left(\begin{array}{lll}0 & 2 & 2\end{array}\right)$ and then triangulating using the $\left(\begin{array}{lll}0 & 0 & l\end{array}\right)$ values, or by measuring a $\left(\begin{array}{lll}2 & 0 & 0\end{array}\right) /\left(\begin{array}{lll}0 & 2\end{array}\right.$ $0)$ type reflection using grazing incidence diffraction.

We have measured the temperature dependence of both out-of-plane $c$ of all samples and in-plane lattice parameters of some samples. Fig. [5] shows the comparison between the lattice constants of films and STO bulk, as well as the LAO substrate. We believe the difference in $(2$ $00)$ and $\left(\begin{array}{lll}0 & 2 & 0\end{array}\right)$ is due to experimental error. It is immediately clear that the lattice parameters evolve smoothly from low temperature up to room temperature, without showing any indication of the structural phase transition. The in-plane lattice constants follow that of the LAO substrate, which implies that the substrate plays the controlling role here.

We attribute the smooth variation of the lattice parameters to the constraint applied by the epitaxy of the film on the far thicker substrate. This substrate clamp-
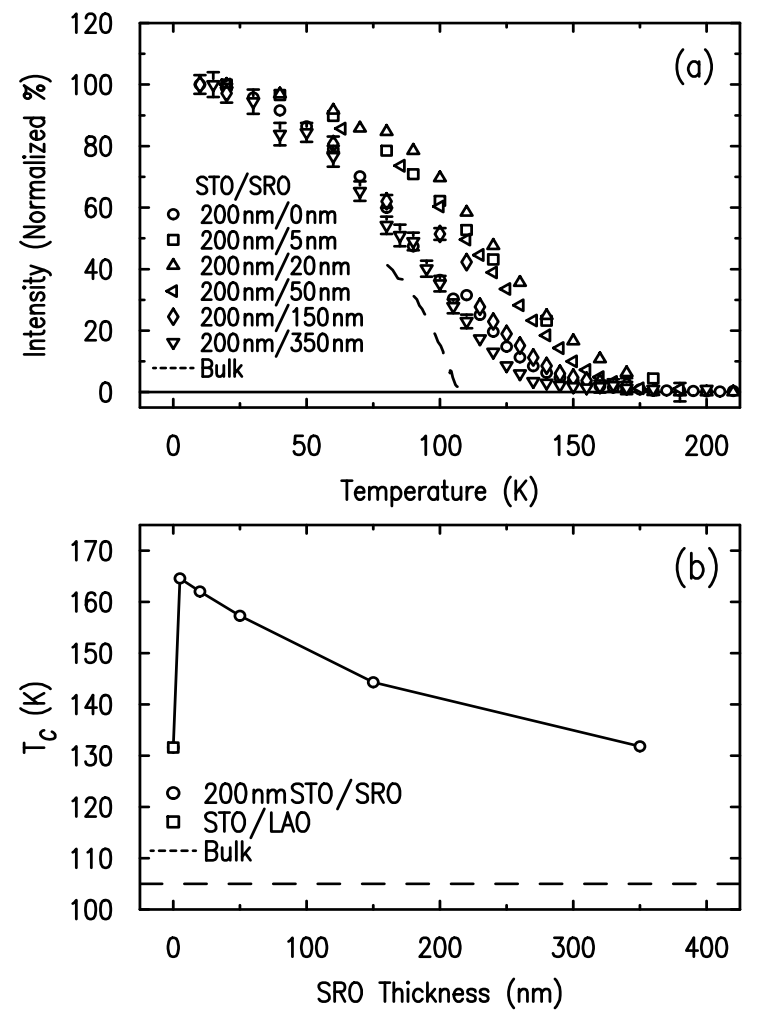

FIG. 4: Temperature dependence of superlattice peak intensity on samples with different SRO thickness. Bulk value from Ref. 19.

ing effect forces the in-plane lattice parameters of films to follow that of substrates. Therefore, even above the phase transition temperature, as defined by intensity of superlattice peaks, STO still has different in-plane and out-of plane lattice constants. In fact, the symmetry of the films at high temperature is no longer cubic, but highsymmetry tetragonal, possibly $\mathrm{P} 4 / \mathrm{mmm}$. In other words, now the phase transition is no longer from cubic Pm $\overline{3} \mathrm{~m}$ to tetragonal $\mathrm{I} 4 / \mathrm{mcm}$, but from high symmetry tetragonal to lower symmetry tetragonal. In bulk STO, the splitting of the lattice parameters at $T_{C}$ results in domain formation and thus lattice relaxation. However, in films, there is no change in the shape of the unit cell during the transition. Therefore, externally, the films always appear as in single domain, and the phase transitions occur without lattice relaxation. Obviously the distorted unit cell favors the $\mathrm{TiO}_{6}$ rotation, so the starting point of the rotation, $\mathrm{T}_{C}$, is much higher in films. Origins of this effect are discussed more fully in Section IV]

\section{B. $\mathrm{NdNiO}_{3}$}

Bulk $\mathrm{NdNiO}_{3}$ is different from bulk $\mathrm{SrTiO}_{3}$ in that it shows discontinuity in temperature dependence of unit cell volume and lattice parameters across the first-order MI transition. ${ }^{21}$ To understand how they behave in films 

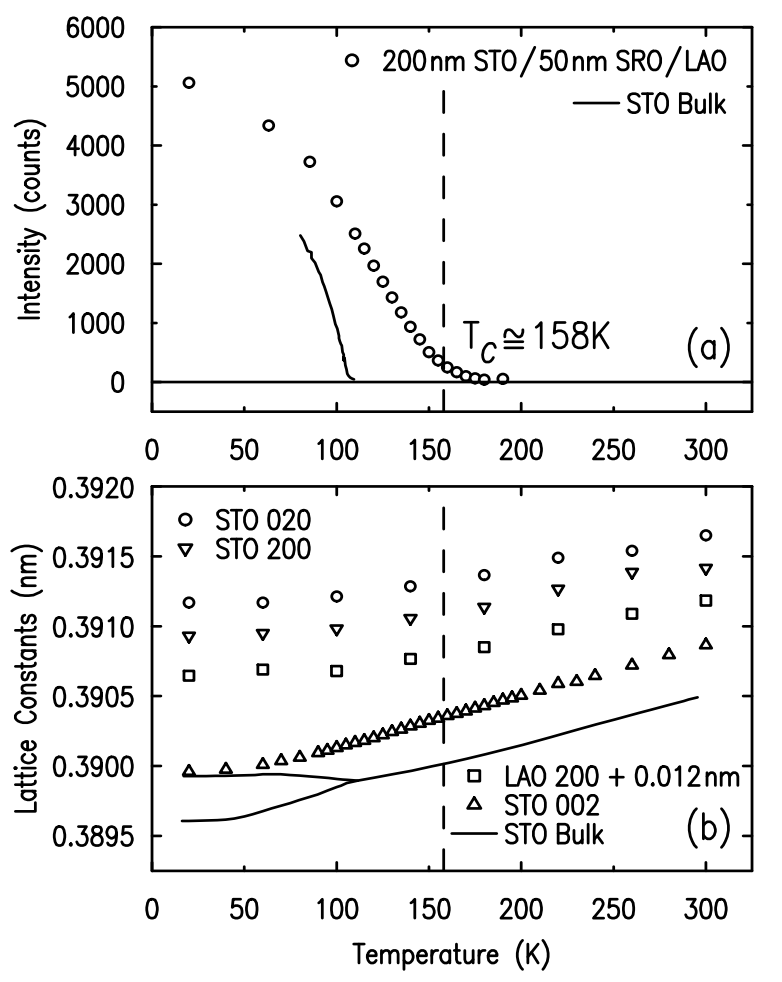

FIG. 5: Temperature dependence of superlattice peak intensity, in-plane and out-of-plane lattice parameters of STO films. Dashed lines indicate $\mathrm{T}_{C}$. LAO and STO bulk data ${ }^{20}$ are shown for comparison.

under the influence of strong substrate clamping effect, we have measured the temperature dependence of inplane and out-of-plane lattice parameters, as well as resistivity, of NNO films.

As shown in Fig. [6 for the $60 \mathrm{~nm}$ sample, the in-plane lattice parameters show the same behavior as in STO films, following closely with the LAO substrates. But the out-of-plane lattice parameters exhibits a jump at the MI transition, although LAO shows nothing anomalous around this range. From Fig. 7 we can see that the discontinuous volume change occurs at about $140 \mathrm{~K}$ in $60 \mathrm{~nm}$ films during warming up, in contrast to $200 \mathrm{~K}$ in bulk. This is in good agreement with the $\mathrm{T}_{C}$ obtained by resistivity measurements (Fig. 8 ). The $200 \mathrm{~nm}$ sample behaves similarly, and its $\mathrm{T}_{C}$ is about $135 \mathrm{~K}$ during cooling down. Another important feature in films is that the volume expansion with respect to the volume on the metallic side is only $0.053 \%$ for $60 \mathrm{~nm}$ sample, considerably smaller than the bulk value of $0.23 \%$. The thermal expansion coefficient $\alpha$ behaves similar in bulk and films.

In bulk NNO, the changes in all three lattice constants contribute to the overall volume discontinuity, while in epitaxial films, this is no longer feasible, since the inplane lattice parameters are constrained by the underlying substrates. Assuming a volume change is necessary for MI transition, then in thin films, the only possible way is to let out-of-plane lattice constant $c$ change ac-

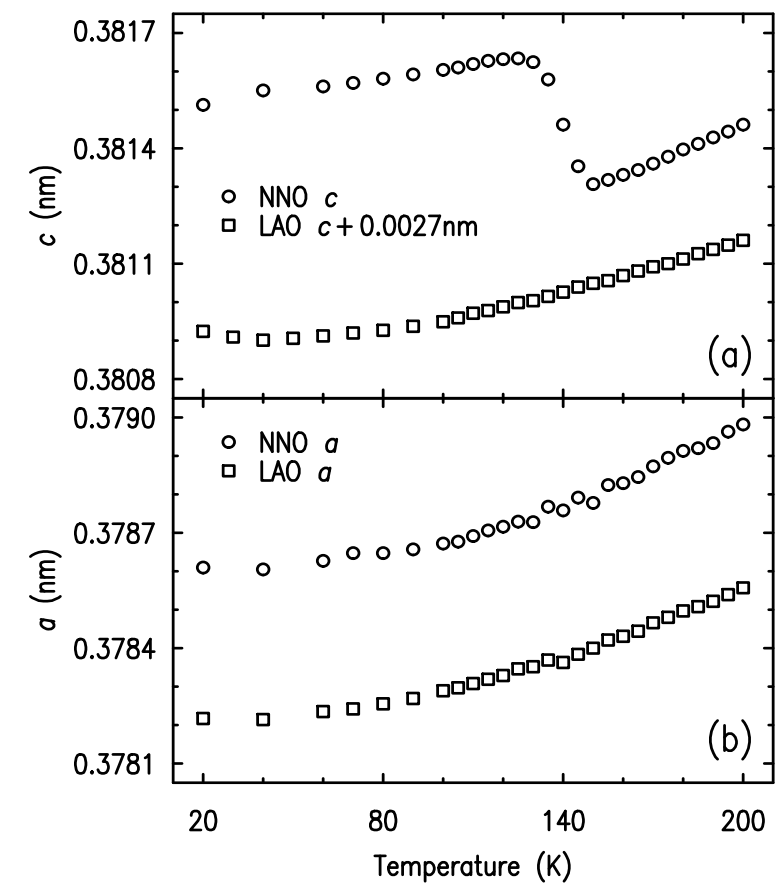

FIG. 6: Out-of-plane and in-plane lattice parameters of $\mathrm{NdNiO}_{3}$ films and LAO substrates during heating up, shown in pseudo-cubic notation.

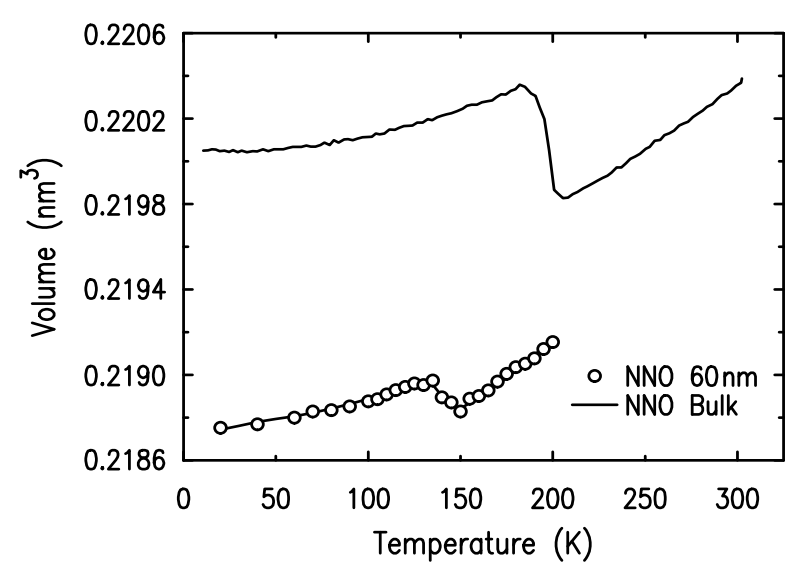

FIG. 7: Temperature dependence of $\mathrm{NdNiO}_{3}$ unit cell volume. Bulk data from Ref. 21.

cordingly. Therefore, in phase transitions with volume discontinuity, the substrate clamping effect still controls the behavior of in-plane lattice parameters, while the outof-plane parameters adapt to accommodate the volume change necessary for the phase transition.

\section{C. $\mathrm{SrRuO}_{3}$}

Bulk $\mathrm{SrRuO}_{3}$ undergoes phase transitions at several temperatures. Above $950 \mathrm{~K}\left(677^{\circ} \mathrm{C}\right)$, $\mathrm{SRO}$ is cubic with $\mathrm{Pm} \overline{3} \mathrm{~m}$ symmetry. Between $950 \mathrm{~K}$ to $820 \mathrm{~K}$ it is tetragonal $\mathrm{I} 4 / \mathrm{mcm}$. Below $820 \mathrm{~K}$, the unit cell becomes or- 


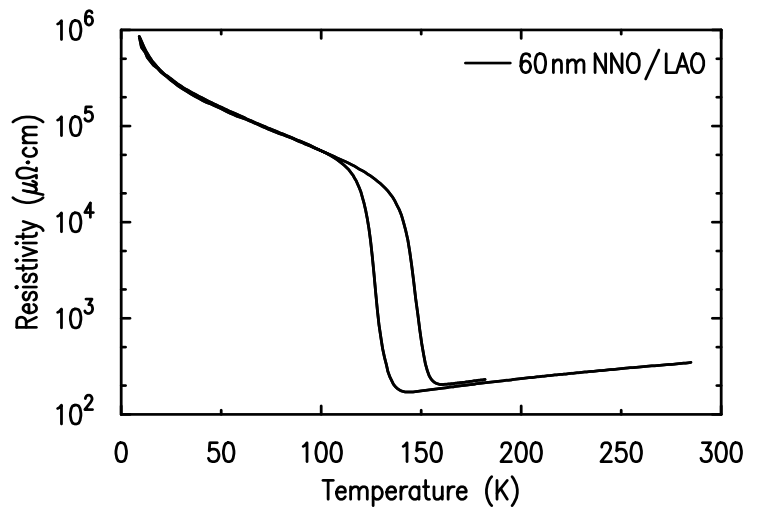

FIG. 8: Resistivity of $\mathrm{NdNiO}_{3}$ films.

thorhombic with space group Pbnm. ${ }^{22}$ At about $160 \mathrm{~K}$, SRO goes through another transition to a ferromagnetic phase.

We found domain structure in the $\mathrm{SrRuO}_{3}$ buffer layers at room temperature, presumably due to tetragonalorthorhombic transition. This appears to be a countercase to the clamping effect we mentioned before. Notice that we will use orthorhombic notation for SRO, but still employ the pseudo-cubic notation for LAO. SRO has a orthorhombic unit cell of about $\sqrt{2} a \times \sqrt{2} a \times 2 a$, where $a$ is pseudo-cubic axis.

The growth temperature $720^{\circ} \mathrm{C}(\sim 993 \mathrm{~K})$ is substantially higher than the first phase transition temperature of SRO. Therefore at growth temperature, SRO is possibly cubic, with lattice mismatch fully relaxed by forming misfit dislocation. Upon cooling down to room temperature, it may form poly-domain structure to relax the strain. 23.24 According to the lattice parameters of pseudo-cubic LAO and orthorhombic SRO, there are six possible domain orientations. ${ }^{25}$ With long axis $(c)$ of SRO unit cell parallel to the $\left[\begin{array}{lll}1 & 0 & 0\end{array}\right]_{c}$ of LAO lattice, we have a pair of 90 degree twin domains, $A$ and $A^{\prime}$. Similarly, with long axis parallel to $\left[\begin{array}{lll}0 & 1 & 0\end{array}\right]_{c}$ of LAO defines $B$ and $B^{\prime}$ domain pair, and parallel to $\left[\begin{array}{lll}0 & 0 & 1\end{array}\right]_{c}$ of LAO defines $C$ and $C^{\prime}$ domains.

We examined SRO layers in several samples by $\mathrm{x}-$ ray reciprocal space mapping at room temperature. At pseudo-cubic $(113)_{c}$ position, which would be $(206)_{o}$ for c domain or $(422)_{o}$ for a(b) domains, we observed two SRO peaks splitting along the cubic $[110]_{c}$ direction. This arrangement of the two peaks suggests $c$ domains, with SRO $c$ axis perpendicular to the surface of the films. The two peaks correspond to $(206)_{o}$ of one domain and $(026)_{o}$ of the other domain from a $90^{\circ}$ twin structure.

We have performed temperature scans from $300 \mathrm{~K}$ to $700 \mathrm{~K}$ on a $5 \mathrm{~nm}$ STO / $350 \mathrm{~nm}$ SRO film on LAO sample, Fig. 9] Both $h k$ plane and $k l$ plane were scanned. At room temperature, there are clearly two SRO peaks. From $500 \mathrm{~K}$ the peaks began to get closer, and finally merged at about $600 \mathrm{~K}$. Upon cooling down, the peak splits at the same temperature, showing a reproducible phase transition. This $\mathrm{T}_{C}$ is almost $150 \sim 200 \mathrm{~K}$ below the
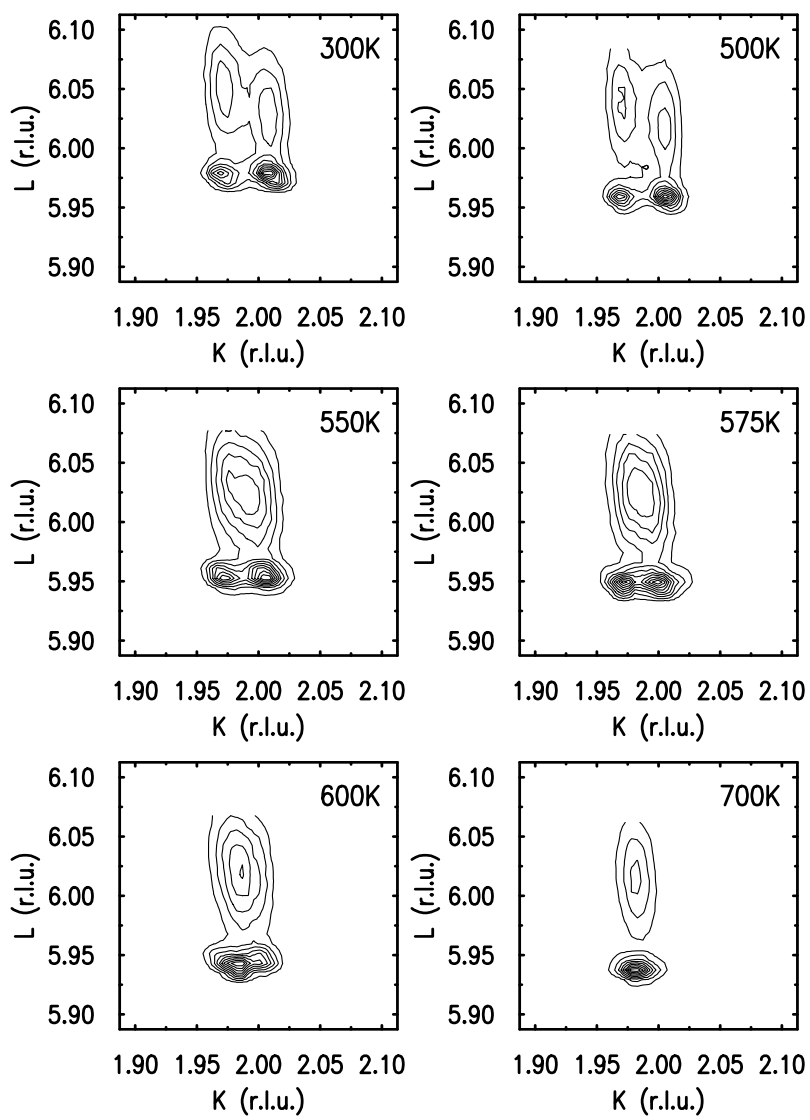

FIG. 9: Reciprocal space mapping shows SRO forming domain structure. The lower peaks are SRO $\left(\begin{array}{lll}2 & 0 & 6\end{array}\right)_{o}$ and $\left(\begin{array}{lll}0 & 2 & 6\end{array}\right)_{o}$. The upper weak peaks are from STO $\left(\begin{array}{lll}1 & 1 & 3\end{array}\right)_{c}$. The unit is in reciprocal lattice unit of SRO.

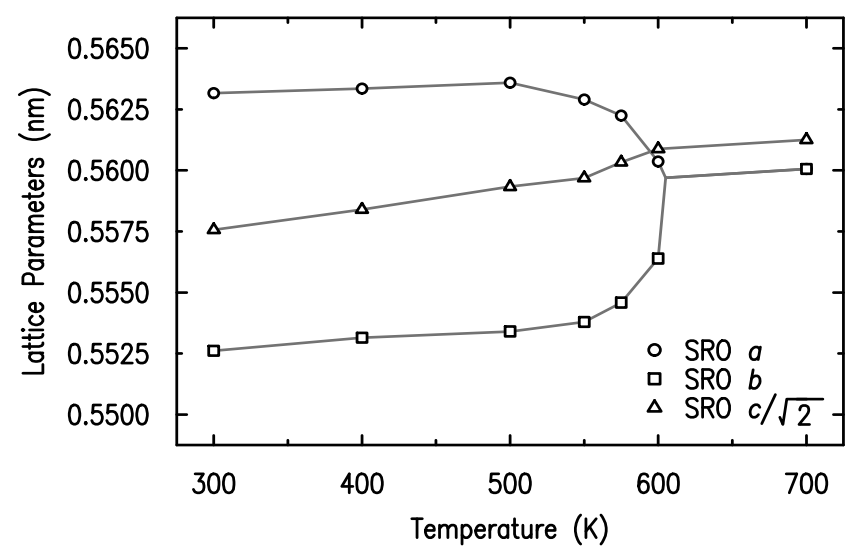

FIG. 10: Temperature dependence of SRO lattice parameters.

bulk value. The temperature dependence of lattice parameters obtained from above measurements are shown in Fig. 10] Overall it is similar to the bulk, though the splitting is bigger. 


\section{DISCUSSION}

\section{A. Order Parameters}

According to Landau theory, the phase transitions of the second order can be quantitatively described by properly defined order parameters, which represent the changes in the symmetry in the crystals when they pass through the phase transition points. The rotational angle of the $\mathrm{TiO}_{6}$ octahedra, $\varphi$, has been identified as the order parameter of phase transition in $\mathrm{STO}_{2}^{27}$ and denotes the deviation from the perfect cubic perovskite structure. The intensity of the superlattice peak is proportional to the square of order parameter. For bulk STO samples, above the phase transition temperature $\mathrm{T}_{C}$, the structure is in cubic symmetry, so there is no superlattice peak. Below $\mathrm{T}_{C}$, the $\mathrm{TiO}_{6}$ octahedra rotate by an angle $\varphi$, and superlattice peaks appear. Concurrently, the three lattice parameters are no longer equal, with $\mathrm{c}$ axis longer than the other two. The changes of the lattice parameters indicates the tetragonality of the unit cell changes at $\mathrm{T}_{C}$. Therefore, we can use the tetragonality as a secondary order parameter to describe this phase transition ${ }^{28.29} \mathrm{We}$ define the tetragonality as:

$$
\gamma=\frac{c-\frac{\left(a_{1}+a_{2}\right)}{2}}{\frac{\left(a_{1}+a_{2}+c\right)}{3}}
$$

where $a_{1}, a_{2}$ and $c$ are the lattice constants in pseudocubic notation, and the average of two in-plane values $a_{1}$ and $a_{2}$ is used to account for possible experimental error. For the cubic phase, $\gamma$ is zero. The tetragonal phase has either positive or negative $\gamma$ depending on if $c$ is greater than or less than $a$. It is interesting to notice that the order parameter defined by rotation angle represents a internal change within the unit cell, while the order parameter obtained from tetragonality gives the external change in the shape of the unit cell.

From above results, we see an important change in the nature of this phase transition in thin films vs bulk. In bulk STO, the internal and external signals are intimately related, the $\mathrm{TiO}_{6}$ octahedra start to rotate while the shape of the unit cell distort at $\mathrm{T}_{C}$. Both signatures show sharp transition points at the same transition temperature of $105 \mathrm{~K}$. In epitaxial thin films, the external lattice shape is no longer free to change, however, the $\mathrm{TiO}_{6}$ octahedra still begin to rotate at particular temperature within this fixed cage. Therefore, although both the order parameters as defined before are good for bulk, in thin films only the internal order parameter can clearly indicate the phase transition. The tetragonality order parameter shows no phase transition, as illustrated in Fig. 11] In other words, the internal degree of freedom is now decoupled from the external degree of freedom.

This phenomenon is not unique for the STO system. Similar cases happen in ferroelectric phase transitions in epitaxial $\mathrm{BaTiO}_{3}$ or other thin films. When bulk $\mathrm{BaTiO}_{3}$ goes through a ferroelectric phase transition at $120^{\circ} \mathrm{C}$, its lattice constants change abruptly, while the unit cell vol-

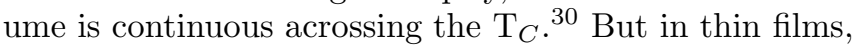
there is no obvious change in all three lattice parameters at the phase transition ${ }^{31.32 .33}$ However from the dielectric measurement, Yoneda and coworkers observed ferroelectricity in the low-temperature phase and paraelectricity in the high-temperature phase in films. This indicates that there is a phase transition with respect to the internal displacement of the $\mathrm{Ti}$ and $\mathrm{O}$ atoms, although the external structural component of the phase transition is suppressed in the presence of epitaxial strain and substrate constraint.

For BTO, the primary order parameter is the spontaneous polarization $\mathbf{P}(\mathbf{D}=0)$, which is proportional to the internal relative displacement of $\mathrm{Ti}$ and $\mathrm{O}$ atoms (the ferroelectricity of the material), and again the tetragonality of the unit cell is the secondary order parameter ${ }^{34} \mathrm{We}$ will see exactly the same relationship between the bulk and thin films as the case of STO. Therefore, from the view point of order parameters, we have a new kind of phase transition which is not possible in bulk.

\section{B. Domain Formation}

It is natural to ask why some films show bulk-like relaxation of lattice parameters at the transitions while others do not. This is equivalent to asking why some films form domains while others do not. We give a qualitative explanation from an energetic point of view.

If there is a material with a cubic to tetragonal phase transition, above $T_{C}$, cubic phase is energetically favored, and below $\mathrm{T}_{C}$ tetragonal symmetry is favored. During the transition, some atoms within the material will have slight displacement, from $X_{\text {film }}$ positions in cubic phase to $X_{\text {film }}^{\prime}$ positions in tetragonal phase. In other words, the potential energy minimums for these atoms shift, and this is the internal driving force for the phase transition and domain formation. If external forces are applied to keep these atoms stay at $X_{\text {film }}$ positions even below $\mathrm{T}_{C}$, the material is strained and the total free energy increased.

On the other hand, imagine we have a completely flexible epitaxial film growth on a substrate, the film atoms would always align with the underlying substrate atoms. The substrate creates a potential energy minimum at each of the $X_{s u b}$ positions directly above the substrate atoms, and these minima only move with substrate. If we need to move some atoms away from these $X_{s u b}$ positions, we must supply some energy. And this again raises the total free energy of the system.

Combining these two pictures, we can see there are two competing energies during this process, involving internal strain energy from film and interface strain energy due to substrate. For a thick film, at the growth temperature, the two minimum $X_{f i l m}$ and $X_{s u b}$ are aligned with each other through formation of misfit dislocations. When the temperature is below $\mathrm{T}_{C}$, the film has the tendency to 

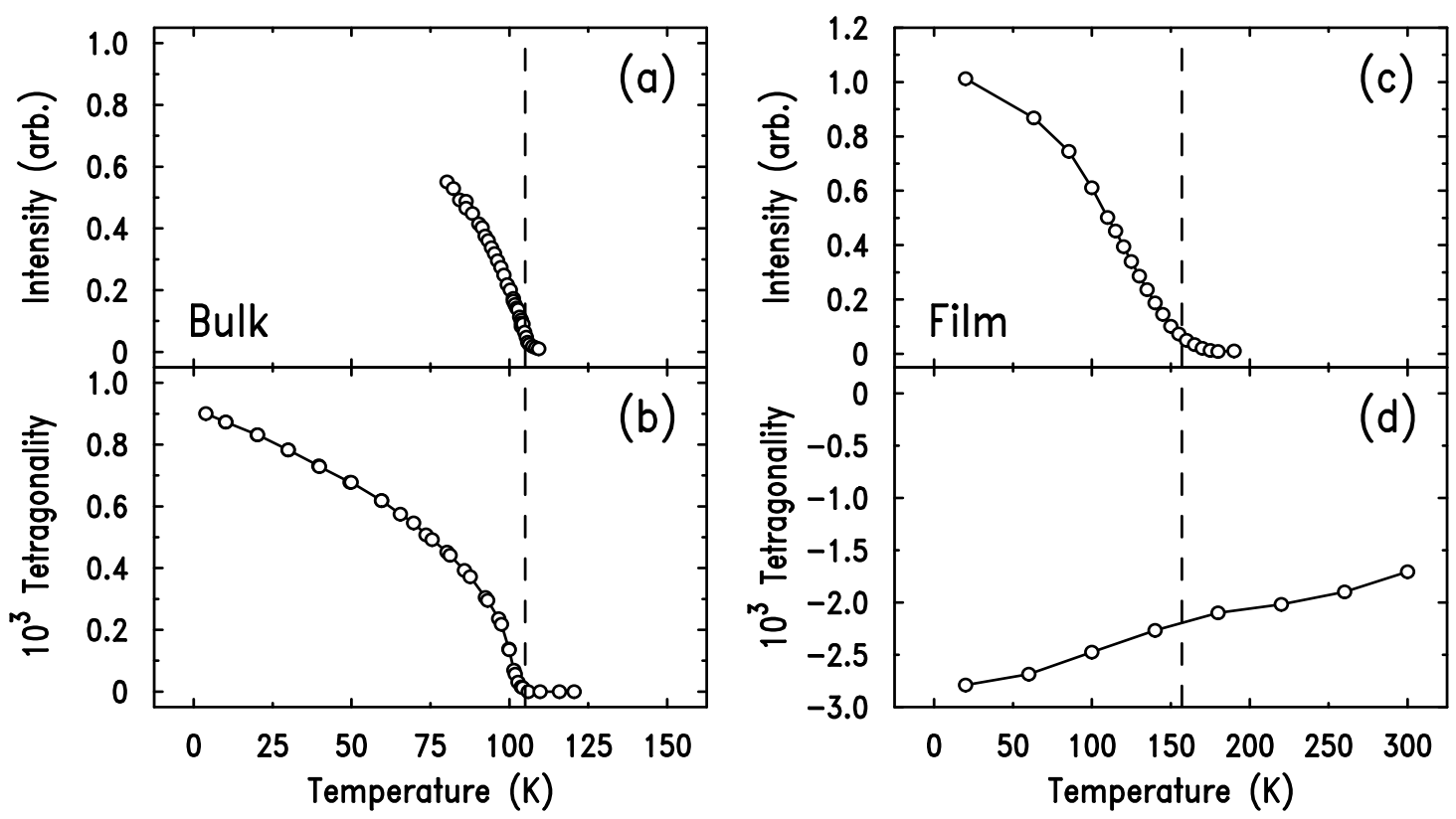

FIG. 11: Order parameters for phase transitions in STO bulk and films, (a) superlattice peak intensity in bulk, (b) tetragonality in bulk, (c) superlattice peak intensity in films, (b) tetragonality in films. Dashed lines indicate $\mathrm{T}_{C}$. Bulk data from Ref. 1926.

complete the structural phase transition, and this makes the two potential energy minima separated into $X_{f i l m}^{\prime}$ and $X_{s u b}$. The deviation of an atom from one minimum will raise the corresponding strain energy, and we expect the increase to be small if the displacement is small.

Generally the structure change is minor when temperature is near $\mathrm{T}_{C}$, so just below $\mathrm{T}_{C}$ the potential minimum at $X_{\text {film }}^{\prime}$ due to film is likely to be close to $X_{\text {sub }}$ position and possibly shallow than $X_{\text {sub }}$. Therefore the driving force for the domain formation is small and the film atoms will still follow substrate. No domain forms. When temperature is considerably lower than $\mathrm{T}_{C}$ and the depth of minimum at $X_{\text {film }}^{\prime}$ exceeds that at $X_{\text {sub }}$, the stable position moves to $X_{\text {film }}^{\prime}$, therefore forming domain is energetically favored. However there will be a barrier between the two minima, and in order to overcome the barrier to reach $X_{\text {film }}^{\prime}$, the atoms need some thermal energy.

For STO, there can be two scenarios, (i) since the structural change is subtle, the potential minimum at $X_{\text {film }}^{\prime}$ may always be shallower than that at $X_{s u b}$; (ii) since the $\mathrm{T}_{C}$ is far below the growth temperature, even if the film has a tendency to form domain below $\mathrm{T}_{C}$, the thermal energy of the film atoms is too low to overcome the barrier to the new position. So even though the minimum at $X_{f i l m}^{\prime}$ is lower, the atoms still stay at the minimum $X_{\text {sub }}$ defined by the substrate. In both cases, it appears macroscopically that the film is frozen to the substrate, without any obvious in-plane lattice change. The domain formation is suppressed by the substrate constraint. $\mathrm{BaTiO}_{3}$ and $\mathrm{NdNiO}_{3}$ films fall into exactly the same situations.

For SRO, the phase transition occurs at above $820 \mathrm{~K}$ in bulk. If we assume the film has the tendency of a phase transition at a similar temperature, then the atoms still have substantial thermal energy, or mobility, to move around. If at a certain temperature the move from $X_{\text {sub }}$ to $X_{\text {film }}^{\prime}$ is energetically favorable, the film atoms will move to the minimum at $X_{\text {film }}^{\prime}$ defined by the internal strain energy, then new domains form. Further experiments are on going to confirm this explanation.

\section{Lattice Parameters vs Thickness}

From XRD measurements of lattice constants, we can see that both film thickness and buffer layer thickness influence the strain state of the STO films. Fig. 12 and Fig. 13 show both the out-of-plane and in-plane lattice parameters at room temperature for different film / buffer combinations. For films grown on the same substrates and buffer layers, when STO film is very thin, the misfit strain is not fully relaxed, so the in-plane lattice constants are expanded, while the out-of-plane lattice constant is reduced in order to keep roughly the same unit cell volume. As the STO thickness increases, the strain within the STO films becomes more and more relaxed, so both the out-of-plane and in-plane lattice parameters move towards the equilibrium bulk values. The thickest film, the $1000 \mathrm{~nm}$ STO / $350 \mathrm{~nm}$ SRO sample, is already indistinguishable from cubic. Considering the experimental error, its three lattice parameters are almost the same. Also it behaves more like bulk crystals, with $\mathrm{T}_{C}$ of $122 \mathrm{~K}$, most close to $105 \mathrm{~K}$.

For the same STO films grown on different SRO layers, the variation of STO lattice constants becomes more complicated. Since the lattice constants of SRO are 


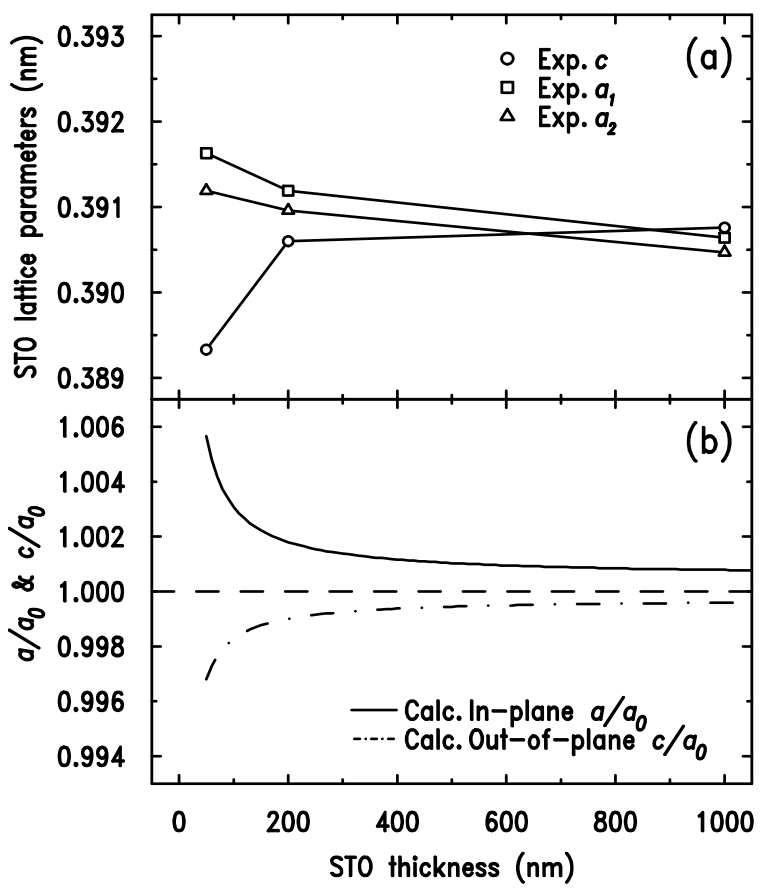

FIG. 12: Lattice parameters for STO films of different thickness on $350 \mathrm{~nm}$ SRO layers. Both experiment data (a) and calculated value (b) are shown. From Ref. 16

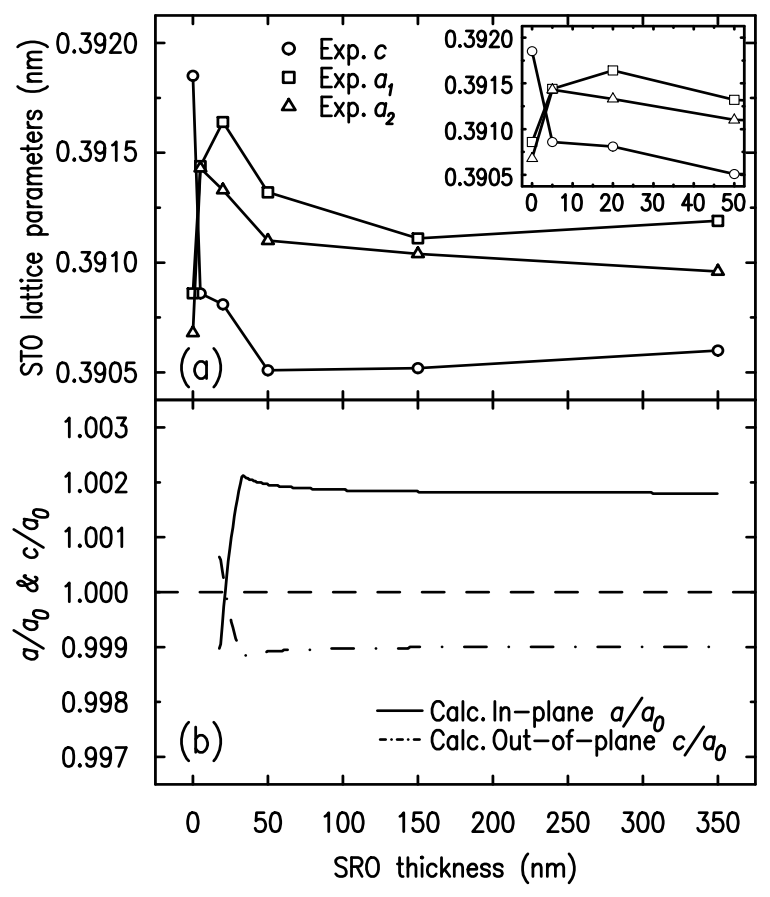

FIG. 13: Lattice parameters for $200 \mathrm{~nm}$ STO films on SRO buffer layers of varying thickness. (a) experiment results, inset is the detail of thin SRO region, (b) calculated STO lattice parameters. From Ref. 16 larger than that of STO, so it seems reasonable to expect thick SRO layers give rise to a larger strain in STO films. But the results turn out to be just opposite, the thinnest SRO layer creates biggest deformation in the STO films. Increasing the SRO thickness, the in-plane lattice parameters in STO films keep decreasing. The out-of-plane lattice constant $\mathrm{c}$ is also decreasing, and always smaller than in-plane ones. An interesting phenomenon is all three parameters are larger than the STO bulk values, which means the volume of the unit cell is bigger than the bulk.

As seen from Fig [13 the STO lattice constants change drastically when SRO thickness goes from $0 \mathrm{~nm}$ to about $20 \mathrm{~nm}$. If STO is grown directly on LAO substrate, the two in-plane lattice parameter $a_{1}$ and $a_{2}$ are smaller than out-of-plane $c$, however, they are still larger than the bulk values. That is to say, STO is expanded by LAO, which is contradictory to the common thought. This indicates that the strain states in the films also rely on other factors, such as the differences between the thermal expansion coefficients of films and substrates, and defects in the films.

To understand the experiment results, we have calculated the dependency of the STO lattice parameters upon the thickness of both STO and SRO layers. The details were published elsewhere ${ }^{16}$ The theoretical model employed takes into account the stress relaxation due to formation of orthorhombic polydomain structure in the SRO buffer layer, as well as the formation of misfit dislocations at the LAO / SRO and STO / SRO interfaces. It has been shown that the internal stress level in films can be controlled using buffer layer that exhibits a structural phase transition.

Taking into account the multiple strain relaxation mechanisms, the in-plane and out-of-plane lattice parameters of STO films as a function of both STO and SRO layer thickness can be calculated, as shown in Fig. 12] and Fig. 13. In general the calculations is in good agreement with experiment data.

\section{Phase Diagram}

It is well known that $\mathrm{T}_{C}$ in films change with different film thickness or different substrates. Generally, one would like to parameterize the control by a simple coordinate and construct an appropriate phase diagram. The most commonly discussed parameter for films is strain. Another candidate for the STO phase transition might be tetragonality at room temperature. Thus we would like to verify a Temperature-Strain phase diagram and a Temperature-Tetragonality phase diagram.

The phase transitions in strained $\mathrm{SrTiO}_{3}$ epitaxial films have been theoretically calculated and discussed by Pertsev et al ${ }^{1}$ The misfit strain-temperature phase diagram they predicted shows that, except for the hightemperature tetragonal phase (HT) being the distorted prototypic cubic phase, there are two purely structural 
tetragonal and orthorhombic states (ST and SO) at lower temperature, and the phase transition temperature is strongly related to the misfit strain. The minimum of $\mathrm{T}_{C}$ is at $105 \mathrm{~K}$ with zero misfit strain, while both positive strain and negative strain increase $\mathrm{T}_{C}$.

Since there is no indication of the structural phase transitions with respect to the changes in lattice constants, we obtained the phase transition temperatures by monitoring the superlattice peaks, which corresponds to the rotation of the $\mathrm{TiO}_{6}$ octahedra. In Fig. 14(a), we plot the relationship between $\mathrm{T}_{C}$ of STO films vs in-plane strain. The general rule for effect of in-plane strain on $\mathrm{T}_{C}$ is that, the larger the in-plane strain, the higher $\mathrm{T}_{C}$. The strain in our samples ranges from $0.01 \%$ to about $0.25 \%$. In this range, the trend of our measurements are in general agreement with the calculations. The phase transition temperatures increase with increasing in-plane strain. Above the transition temperature, the STO film is a high-symmetry tetragonal phase due to the biaxial strain and substrate constraint. However, there are some differences. They predicted that $\mathrm{T}_{C}$ would increase very slowly for tensile strains, not higher than $115 \mathrm{~K}$ for $2 \%$ strain. But our results show remarkably larger increases in $\mathrm{T}_{C}$ even for much smaller strains, about $160 \mathrm{~K}$ for $0.25 \%$ strain. In addition, it is possible to induce the same amount of in-plane strain in STO films by varying either STO thickness or SRO layer thickness. However the transition temperatures are different for these two cases, thus $\mathrm{T}_{C}$ vs strain is not a simple monotonic function.

From Fig. 14(b) $\mathrm{T}_{C}$ vs tetragonality of STO unit cell, we also found there is no simple relationship between them. The main factor influencing the tetragonality is the thickness of STO itself. The thin STO films tend to have high tetragonality while thick STO films is more bulk-like with tetragonality close to 0 . With the same STO thickness of $200 \mathrm{~nm}$, the films on different SRO layers have almost the same tetragonality. However the $\mathrm{T}_{C} \mathrm{~s}$ of these films are quite different. That is to say, tetragonality is not an adequate factor to dictate $\mathrm{T}_{C}$. This is also consistent with fact that the tetragonality of each film does not change across the phase transition.

Some reasons for the discrepancy between theory and experimental data may include: (i) an ideal cubic substrate was used for calculations, but the real buffersubstrate systems usually have mosaic structures and domains. This leads to inhomogeneous strain conditions across the films; (ii) the different definition of $\mathrm{T}_{C}$ we used may give a slightly higher $\mathrm{T}_{C}$. However, we believe the primary reason is because film systems are inherently more complicated than the model proposed. The strain state in a film is due to more than the difference between the lattice parameters of film and substrate. On one hand, two strain relaxation mechanisms, forming misfit dislocations at the film-substrate interface and forming domain structures within the films, greatly reduce the actual misfit strain comparing with that calculated by respective bulk values. On the other hand, the differ-
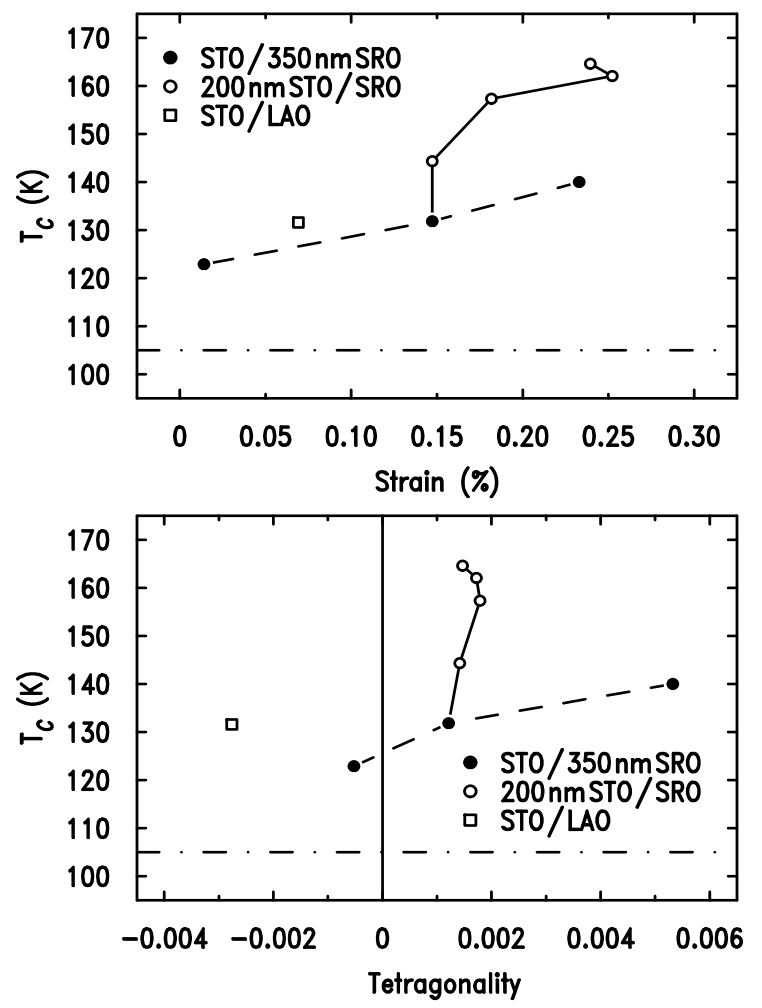

FIG. 14: Dependence of $\mathrm{T}_{C}$ on (a) in-plane strain, (b) tetragonality

ence between the thermal expansion coefficients of films and substrates, together with substrate constraint, create another kind of strain, which depends on temperature, but not on the lattice constants of films and substrates. Furthermore, non-equilibrium defects increase unit cell volume, 35.36 and thus change strain in a manner different than epitaxial mismatch.

Adding together all these complications, the $\mathrm{T}_{C}$ vs strain is not a simple function in real films, and an equilibrium phase diagram may not be generally applicable.

\section{CONCLUSION}

Through a systematic study of three distinctive film systems, we have a more comprehensive understanding on the phase transitions in epitaxial perovskite films. The final strain state of film, and thus the $\mathrm{T}_{C}$, results from competition between multiple mechanisms, and may not be ascribed to a single parameter, such as misfit strain. There are a few general rules, such as thickness dependence of strain and substrate constraint, which are followed by all film systems. However, a particular film system may possess its own unique features, which must be characterized individually.

We have studied the structural phase transitions in strained STO films grown on LAO substrates with SRO buffer layers. As indicated by the superlattice peaks asso- 
ciated with the lower temperature tetragonal phase, the phase transitions occur at higher temperature in strained films, with larger in-plane strain giving higher $\mathrm{T}_{C}$. However, there is no obvious indication of this transition from temperature dependence of the lattice parameters, and tetragonality is no longer a good secondary order parameter. This is an important change in the nature of this phase transitions. Due to the epitaxial strain and substrate clamping effect, the internal degree of freedom, the rotation of the $\mathrm{TiO}_{6}$ octahedra, decouples from the external degree of freedom. Our data show that both the thickness of STO thin films and SRO buffer layers influence the magnitude of the strain within STO films. The in-plane strain decreases with increasing STO thickness, however, thinner SRO layers give rise to larger in-plane strains in STO films. This result is in good agreement with our calculations. Combining the $\mathrm{T}_{C}$ and strain measurements, it is found that the in-plane strains have considerably larger effect on $\mathrm{T}_{C}$ than previously predicted.

From our results of $\mathrm{NdNiO}_{3}$ films grown on LAO, it is much clearer that substrate constraint is an important parameter for epitaxial films. The in-plane lattice parameters of film are tied down by the underlying substrate, following the trend of substrate through the whole temperature range, without change even across the phase transition in films. This leaves the out-of-plane lattice parameter the only variable to accommodate the vol- ume change during the phase transition. This substrate clamping effect has a profound influence on the strain within film, and can induce a substantial change in phase transition temperature $\mathrm{T}_{C}$.

$\mathrm{SrRuO}_{3}$ films show us even more complications. In this system we observed domain formation at elevated temperature. This means the substrate constraint sometimes may give way to other much stronger strain relaxation mechanism. This phenomenon illustrates that the final strain states in films are the results of interaction between multiple competing mechanisms, such as internal strain energy within films and interface strain energy.

\section{Acknowledgments}

BW thanks the Cottrell Scholar Program of the research corporation for partial support of this work. This material is based upon work supported by the National Science Foundation under Grant No. DMR-0239667 (BW, FH), DMR-0132918 (ZB, PA), and DMR-9702632 (XX). Work at Brookhaven is supported by Division of Material Sciences, U.S. Department of Energy under contract DE-AC02-98CH10886. The work at UConn was also supported by the University of Connecticut Research Foundation.
* Electronic address: fhe@phys.uconn.edu

1 N. A. Pertsev, A. K. Tagantsev, and N. Setter, Phys. Rev. B 61, R825 (2000).

2 N. A. Pertsev, A. G. Zembilgotov, and A. K. Tagantsev, Phys. Rev. Lett. 80, 1988 (1998).

3 A. L. Roytburd, J. Appl. Phys. 83, 228 (1998).

4 Z. G. Ban and S. P. Alpay, J. Appl. Phys. 91, 9288 (2002).

5 X. X. Xi, H.-C. Li, W. Si, A. A. Sirenko, I. A. Akimov, J. R. Fox, A. M. Clark, and J. Hao, J. Electroceramics 4, 393 (2000).

${ }^{6}$ G. Shirane and Y. Yamada, Phys. Rev. 177, 858 (1969).

7 K. A. Müller and H. Burkard, Phys. Rev. B 19, 3593 (1979).

8 H. Uwe and T. Sakudo, Phys. Rev. B 13, 271 (1976).

9 J. Hemberger, M. Nicklas, R. Viana, P. Lunkenheimer, A. Loidl, and R. Böhmer, J. Phys.: Condens. Matter 8, 4673 (1996).

10 J. G. Bednorz and K. A. Müller, Phys. Rev. Lett. 52, 2289 (1984).

11 M. Itoh, R. Wang, Y. Inaguma, T. Yamaguchi, Y.-J. Shan, and T. Nakamura, Phys. Rev. Lett. 82, 3540 (1999).

12 D. Fuchs, C. W. Schneider, R. Schneider, and H. Rietschel, J. Appl. Phys. 85, 7362 (1999).

13 P. C. Canfield, J. D. Thompson, S.-W. Cheong, and L. W. Rupp, Phys. Rev. B 47, 12357 (1993).

14 A. Tiwari, C. Jin, and J. Narayan, Appl. Phys. Lett. 80, 4039 (2002).

15 F. He, B. O. Wells, S. M. Shapiro, M. v. Zimmermann, A. Clark, and X. X. Xi, Appl. Phys. Lett. 83, 123 (2003).

16 Z.-G. Ban, F. He, S. P. Alpay, B. O. Wells, and X. X. Xi,
Appl. Phys. Lett. 84, 000 (2004).

17 H. Li, W. Si, A. D. West, and X. X. Xi, Appl. Phys. Lett. 73, 190 (1998).

18 S. Doi and I. Takahashi, Philo. Mag. A 80, 1889 (2000).

19 S. A. Hayward and E. K. H. Salje, Phase Transitions 68, 501 (1999).

20 A. Okazaki and M. Kawaminami, Mat. Res. Bull. 8, 545 (1973).

21 J. L. García-Muňoz, J. Rodríguez-Carvajal, P. Lacorre, and J. B. Torrance, Phys. Rew. B 46, 4414 (1992).

22 B. J. Kennedy and B. A. Hunter, Phys. Rev. B 58, 653 (1998).

23 S. P. Alpay and A. L. Roytburd, J. Appl. Phys. 83, 4714 (1998).

24 J. C. Jiang and X. Q. Pan, J. Appl. Phys. 89, 6365 (2001).

25 J. C. Jiang, W. Tian, X. Q. Pan, Q. Gan, and C. B. Eom, Appl. Phys. Lett. 72, 2963 (1998).

${ }^{26}$ K. Hirota, J. P. Hill, S. M. Shapiro, G. Shirane, and Y. Fujii, Phys. Rev. B 52, 13195 (1995).

27 K. A. Müller, W. Berlinger, and F. Waldner, Phys. Rev. Lett. 21, 814 (1968).

28 M. Sato, Y. Soejima, N. Ohama, A. Okazaki, H. J. Scheel, and K. A. Müller, Phase Transitions 5, 207 (1985).

29 A. Binder and K. Knorr, Phys. Rev. B 63, 094106 (2001).

30 A. Devonshire, Phil. Mag. 40, 1040 (1949).

31 Y. Yoneda, H. Kasatani, H. Terauchi, Y. Yano, T. Terashima, and Y. Bando, J. Phys. Soc. Japan 62, 1840 (1993).

32 H. Terauchi, Y. Watanabe, H. Kasatani, K. Kamigaki, Y. Yano, and T. Terashima, J. Phys. Soc. Japan 61, 2194 
(1992).

33 S. P. Alpay, I. B. Misirlioglu, A. Sharma, and Z.-G. Ban, J. Appl. Phys. 95, 8118 (2004).

34 R. A. Cowley, Phys. Rev. B 13, 4877 (1976).

35 E. J. Tarsa, E. A. Hachfeld, F. T. Quinlan, J. S. Speck, and M. Eddy, Appl. Phys. Lett. 68, 490 (1996).

36 L. S.-J. Peng, X. X. Xi, B. H. Moeckly, and S. P. Alpay, Appl. Phys. Lett. 83, 4592 (2003). 